\title{
Compression Techniques for Improved Algorithm Computational Performance
}

\author{
Joseph N. Zalameda ${ }^{\mathrm{a}}$, Patricia A Howell ${ }^{\mathrm{b}}$, and William P. Winfree ${ }^{\mathrm{b}}$ \\ ${ }^{a}$ U. S. Army Research Laboratory, Vehicle Technology Directorate \\ Nondestructive Evaluation Sciences Branch MS 231 \\ NASA Langley Research Center 23681 \\ ${ }^{\mathrm{b}}$ Nondestructive Evaluation Sciences Branch MS 231 \\ NASA Langley Research Center 23681
}

\begin{abstract}
Analysis of thermal data requires the processing of large amounts of temporal image data. The processing of the data for quantitative information can be time intensive especially out in the field where large areas are inspected resulting in numerous data sets. By applying a temporal compression technique, improved algorithm performance can be obtained. In this study, analysis techniques are applied to compressed and non-compressed thermal data. A comparison is made based on computational speed and defect signal to noise.
\end{abstract}

Keywords: Quantitative thermal nondestructive evaluation, file compression, improved processing time, principle component analysis and defect signal to noise.

\section{INTRODUCTION}

There is a growing interest in the use of thermal methods for wide area nondestructive evaluation (NDE) because the technology is noncontact, rapid, applicable to complex geometries, and quantitative. The technique is safe, requiring only a small amount of heat be applied to the surface of the structure using a flash or quartz lamp heat source. The technology is becoming more portable for field inspections because of newly developed infrared camera and heat source technologies. The measurement of the surface temperature response is performed remotely using an infrared camera. The inspection field of view is approximately $0.3 \times 0.3$ meters square (around 1 square foot) and because of this large areas can be inspected rapidly.

When inspecting large areas, multiple inspection shots are scanned over the entire surface. The recorded data set per inspection shot consists of a set of images recorded in time with a typical sample rate of 60 Hertz, and therefore each data set can be very large on the order of tens to hundreds of megabytes. For example the inspection of a $1.2 \times 1.8$ meters square area would require a minimum of 24 inspection shots. If one was to assemble the inspection images into one large image this number would be slightly higher because of the required inspection overlap. Some applications may also require inspection of the back side, if there is access, to completely document the integrity of the structure. This would increase the total number of inspection shots by a factor of 2 . The number of inspection shots quickly add up and results in the requirement to process large amounts of data. Improved computational performance can result in significant overall time savings. This would allow the inspector in the field to more quickly obtain the inspection results.

The objective of this paper is to investigate use of data compression techniques to enhance computational speed without significantly decreasing defect signal to noise. Several data compression techniques are studied and include subsampling, fixed window averaging, and variable window or step averaging. The compression algorithm is applied to the data before processing. The data analysis algorithms used in the study for defect detection are model based fitting 
for the measurement of thermal diffusivity and principle component analysis. Model based fitting is commonly used in thermography for the quantitative detection of subsurface defects through the measurement of thermal diffusivity. ${ }^{1}$ Quantitative thermal diffusivity is measured by minimizing the squared difference between the data and a thermal model. Any variations due to this material property can indicate defects. Principle component analysis (PCA) has recently been applied for processing thermal NDE data. ${ }^{2}$ This algorithm is based on decomposition of the thermal data into its empirical orthogonal functions. Since thermal NDE signals are well behaved and slowly decaying waveforms, the dominant spatial variations of the data set is usually contained in the first or second eigenvector. In this study, thermal data is obtained on samples with known defects and the data analysis techniques are applied to compressed and non-compressed data. A comparison is made based on computational speed and defect signal to noise.

\section{DATA COMPRESSION TECHNIQUES}

Three data compression techniques are applied to the thermal data: sub-sampling, fixed window averaging, and variable window or step averaging. The thermal data is obtained by using flash lamps located on the same side as the thermal camera. The infrared camera is a cooled $256 \times 256$ focal plane InSb array operating in the $3-5$ micrometer wavelength band. The acquired data sets are composed of a series of 16 bit digital images captured at 1/60 or 1/30 of a second. Before compression, the data is offset corrected by subtracting a background frame acquired before heating. The compression algorithm is applied to the data, before processing, to increase processing speed.

The first compression algorithm studied was sub-sampling. The data was sub-sampled by selecting every $4^{\text {th }}$ and $8^{\text {th }}$ frame for compression factors of 4 and 8 respectively. This is a basic way to reduce the number of images used for processing and is studied for comparison to the other compression techniques. An example sub-sampled waveform is shown in the right plot of Figure 1 where the measured temperature response is plotted for a given pixel point. In this example, the sub-sampled data was produced by selecting every $8^{\text {th }}$ frame. The original signal is also shown in Figure 1. The next compression algorithm studied is fixed window averaging. The fixed window averaging was performed by averaging a fixed number of frames to reduce the number of data points. The number of frames averaged were 5 , 10 , and 20. The 5 frame averaged waveform is shown in Figure 2. This analysis technique converts the data set from 16 bit integer to 32 bit real data to prevent summing overflow. The last compression technique studied was variable window averaging or step window averaging. This technique involves increasing the window to average images in steps of $2^{\mathrm{n}}$ and applying the window to produce the next $\mathrm{n}+1$ (for $\mathrm{n}=0,1,2,3 \ldots$ ) images in the compressed data set (therefore the series would be $1,2,2,4,4,4,8,8,8,8,16,16,16,16,16 \ldots .$.$) . An example waveform is shown in Figure 2$.

Original Waveform

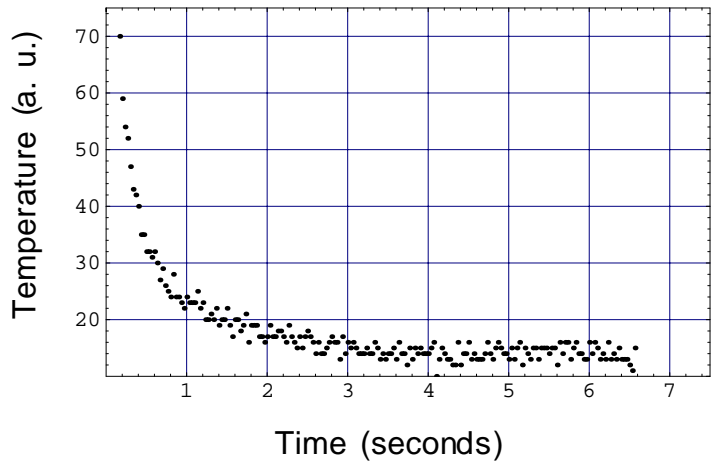

Sub-Sampled Every 8 Points

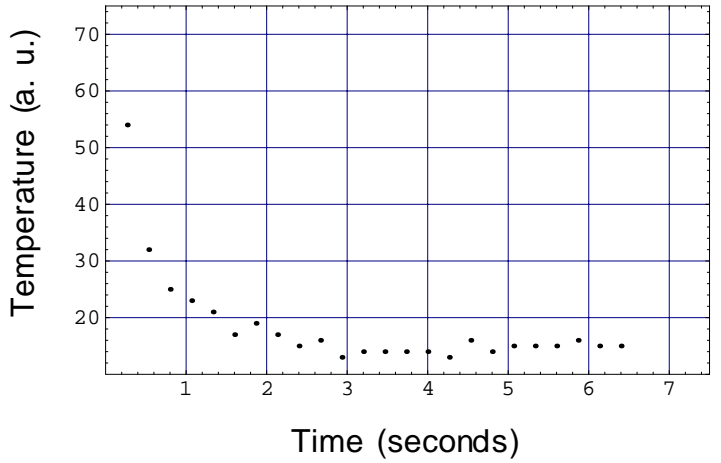

Figure 1. Original and sub-sampled temperature response for a given pixel point in time. 
5 Point Fixed Window Average

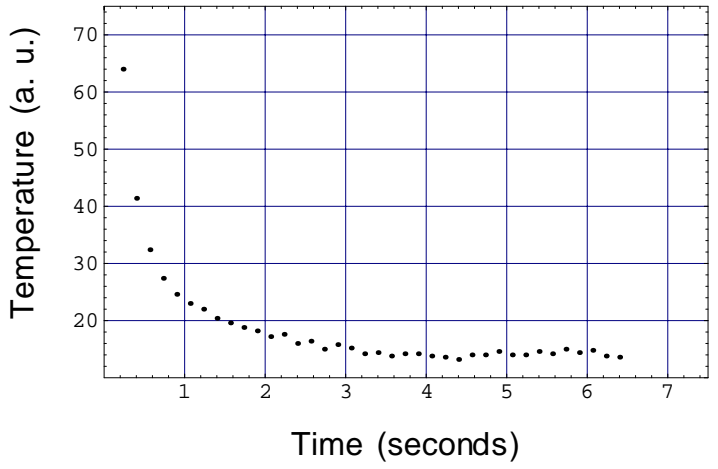

Variable Window Average

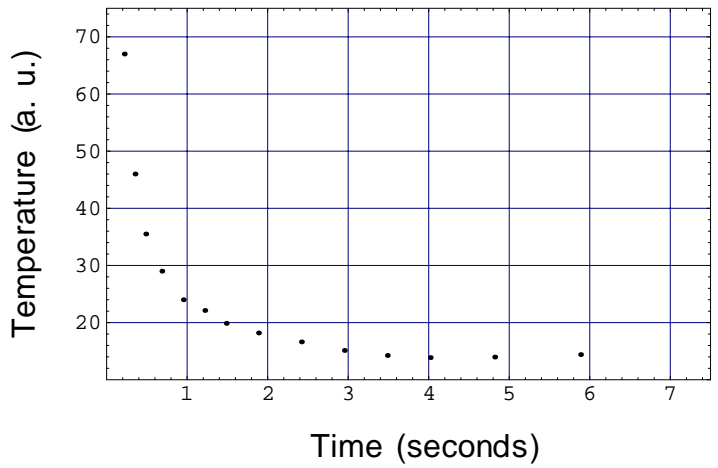

Figure 2. Fixed window averaging (5 points) and variable window averaging temperature response.

\section{SAMPLES STUDIED}

Thermal NDE data was acquired on 2 samples with known defects. The samples were a composite panel and a reinforced carbon carbon (RCC) composite sample. The composite panel contained manufactured delamination defects at different depths. The delaminations were created using a rectangular stamp to form an air gap. The quasi-isotropic composite panel with a lay-up of $[0 /, 45 /, 90 /,-45 /, 0 /, 0,-45,90,45,0]$ was $30.5 \times 30.5$ centimeters in size. The 10 ply panel was 0.20 centimeters thick. The delamination defect areas were square with sizes of $14.5,6.54,3.6$, and 1.6 square centimeters. The inspection was performed on the backside of the sample and therefore the defects were buried at various depths of 50,60,70,80 and 90 percent of the total thickness. The sample configuration is shown in Figure 3. The RCC composite panel contained interior material loss defects of 15, 46, and 85 percent of the total thickness. The average thickness of the sample was 0.69 centimeters. The material loss defects were circular with diameters of 1.3 and 0.65 centimeters. The defect layout of this sample is shown in Figure 4.

\section{$50 \% \quad 60 \% \quad 70 \% \quad 80 \% 90 \%$}

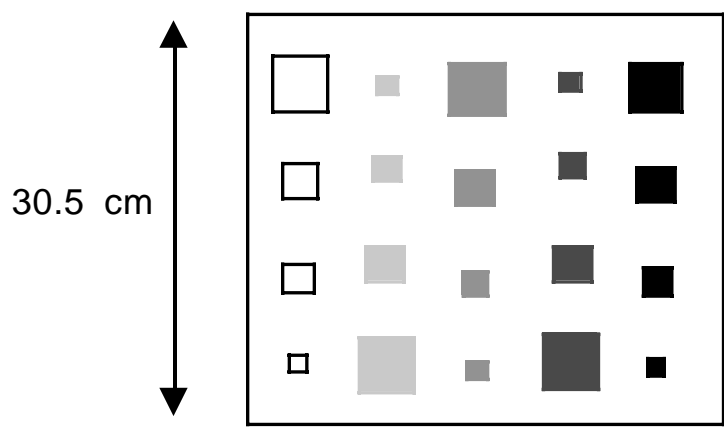

Delamination dept $h$ is percent of thickness. ( $t$ hickness $=$ $0.20 \mathrm{~cm}$ )

Figure 3. Defect layout configuration for composite panel with delamination defects. 


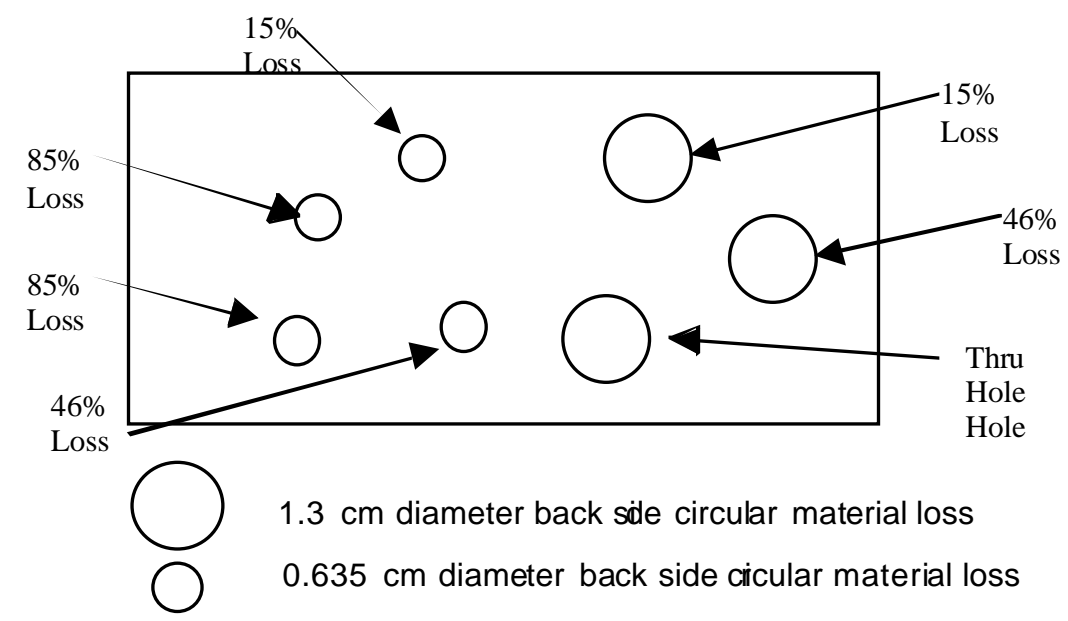

Figure 4. Defect layout configuration for RCC composite sample with material loss defects.

\section{DATA ANALYSIS RESULTS}

The various compression routines were applied to the data before applying the defect detection algorithms. The defect detection algorithms used were model based fitting for thermal diffusivity measurement and PCA analysis. Model based fitting and PCA analysis were applied to the composite panel and RCC sample data respectively.

\subsection{Model Based Reduction on Composite Panel}

Defect contrast can be enhanced by imaging the effective diffusivity of the sample. This data reduction algorithm involves fitting a theoretical 1-dimensional temperature response model with the measured temporal temperature response pixel by pixel. ${ }^{1}$ By fitting a model with the data, noise is reduced. Uneven heating and emissivity variations are also minimized because the fitting is based on the transient portion of the temperature response. Parameters such as final temperature and thermal diffusivity over the thickness squared are varied using a nonlinear fitting algorithm to minimize the squared difference between the model and data. If the thickness is known then the thermal diffusivity can be calculated. The model used for fitting the data is given in equation 1 below as:

$$
\begin{gathered}
T_{S}(\mathrm{t})=\mathrm{T}_{F} \frac{\left(1+2 \operatorname{Exp}\left[\frac{-1^{2}}{\alpha\left(\mathrm{t}-\mathrm{t}_{\text {shift }}\right)}\right]\right)}{\sqrt{\frac{\pi \alpha\left(\mathrm{t}-\mathrm{t}_{\text {shift }}\right)}{1^{2}}}} \text { for } \mathrm{t} \leq 0.62 \frac{1^{2}}{\alpha} ; \text { and } \\
T_{S}(\mathrm{t})=\mathrm{T}_{F}\left(1+\operatorname{Exp}\left[\frac{-\alpha\left(\mathrm{t}-\mathrm{t}_{\text {shift }}\right) \pi^{2}}{1^{2}}\right]\right) \text { for } \mathrm{t}>0.62 \frac{1^{2}}{\alpha} .
\end{gathered}
$$

$T_{S}(\mathrm{t})$ is the surface temperature response, $\mathrm{T}_{F}$ is the final temperature, $\alpha$ is the thermal diffusivity, 1 is the thickness, and $\mathrm{t}$ is time. The single side heating temperature response equation assumes no convection losses and the flash duration is not considered instantaneous. The finite pulse time must be taken into account when doing quantitative measurements. The flash pulse time has been previously measured to be 0.0029 seconds and to compensate for the finite time of the flash pulse the parameter $t_{\text {shift }}$ is used. ${ }^{3}$ Thermal measurements were performed on the composite panel where the inspection parameters were $30 \mathrm{~Hz}$ frame rate and the number of frames grabbed were 200 for a total inspection time of 
6.67 seconds. The various compression routines were applied to the data before the model fitting data reduction algorithm was used. Shown in Figure 5 are the composite panel thermal diffusivity images without compression and sub-sampled every $8^{\text {th }}$ frame. The sub-sampled thermal diffusivity image was $46.8 \%$ faster to compute. Also shown in Figure 6 are the composite panel thermal diffusivity images with fixed window averaging of 20 frames and variable window averaging. The fixed window averaging and the variable window average diffusivity images were $48.4 \%$ and $45.5 \%$ faster (factor of 2 speed increase) to compute respectively.

To facilitate comparison, a horizontal line plot was generated over the first row of defects (around image line 180). The line plots represents the average of 5 horizontal lines and this is shown in Figure 7 for the non-compressed, subsampled every $8^{\text {th }}$ point, and variable window average images. From the averaged line plot, an average SNR value for the first and third defect was calculated by taking the absolute value of the pixels over the defects minus the average of the background pixels and dividing by the standard deviation of the background pixels. The background pixels were obtained by averaging pixels before and after each defect. The average background value was subtracted from the defect value before dividing by the background standard deviation to minimize the effects of the non-flat background. The defect averaged signal to noise values were 5.0, 2.3, 4.4, 4.4 and 4.4 for the non-compressed, sub-sampled every $8^{\text {th }}$ point, 5 point fixed window average, 20 point fixed window average, and variable window average thermal diffusivity images respectively. There is significant degradation in the SNR for the sub-sampled data set and very little degradation for the averaging compression schemes.

\subsection{Principle Component Analysis Reduction on RCC Sample}

PCA is commonly applied for the thermal inspection of RCC. This algorithm is based on decomposition of the thermal data into its principle components or eigenvectors. ${ }^{2}$ Singular value decomposition is a routine used to find the singular values and corresponding eigenvectors of a matrix. Since thermal NDE signals are well behaved and slowly decaying waveforms, the predominant spatial variations of the entire data set are usually contained in the first or second eigenvectors, accounting for most of the data variance. The PCA is computed by defining a data matrix A, where the time variations are along the columns and the spatial image pixel points are row-wise. The matrix A can then be decomposed as:

$$
\mathrm{A}=\mathrm{U}^{*} \Gamma^{*} \mathrm{~V}^{\mathrm{T}}
$$

where $\Gamma$ is the a diagonal matrix containing the singular values, $\mathrm{V}$ is an orthogonal matrix with dimensions corresponding to the number of images or time history, and $U$ is an orthogonal matrix which contains the eigenvectors describing the spatial variations, and therefore, each column of $U$ contains the eigenvectors which can be configured to generate the PCA image. Typically the first eigenvector PCA image provides good contrast for defect detection. Thermal measurements were performed on the RCC sample where the inspection parameters were $60 \mathrm{~Hz}$ frame rate and

Original Diffusivity Image

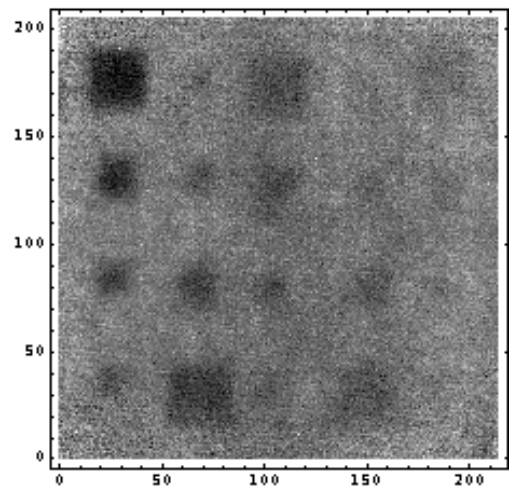

Sub-Sample Every 8 Frames

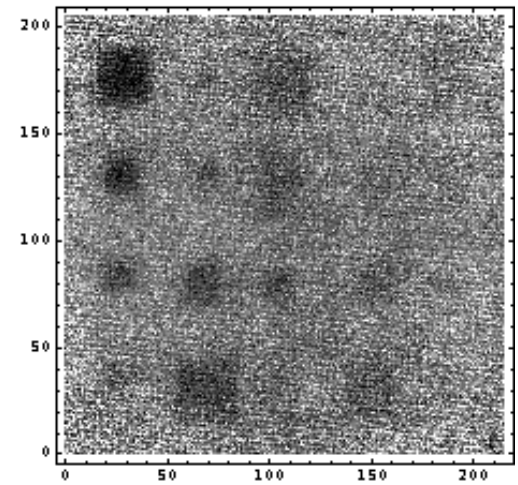

Diffusivity $=$ $0.007 \mathrm{~cm}^{2} / \mathrm{sec}$

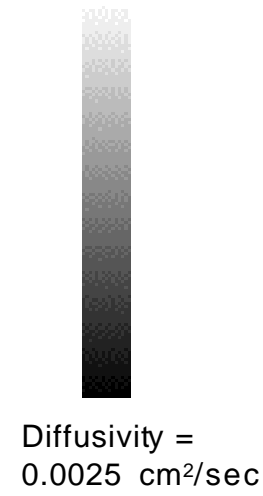

Figure 5. Composite panel thermal diffusivity images without compression and sub-sampled every 8 frames. 
Fixed Window Average 20 Frames

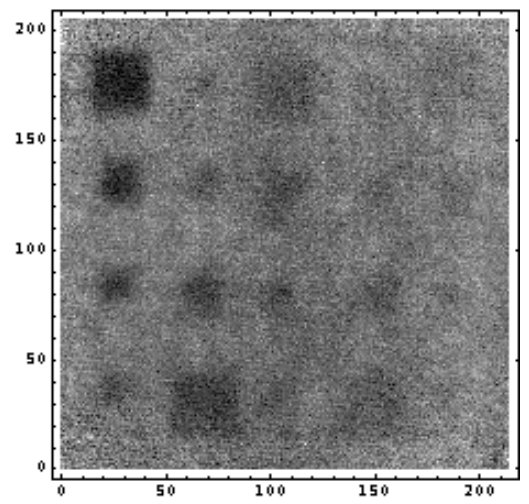

Variable Window Averaging Diffusivity Image

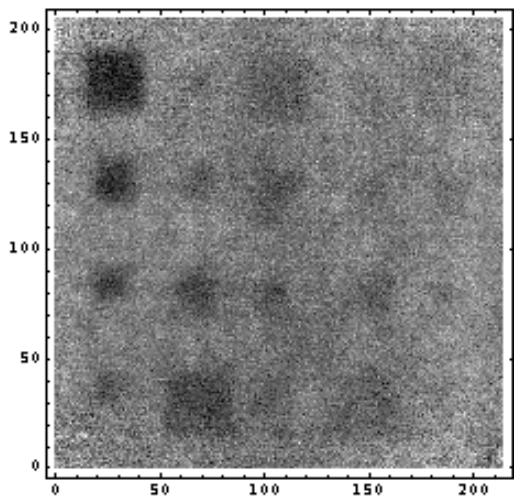

Diffusivity =

$0.007 \mathrm{~cm}^{2} / \mathrm{sec}$

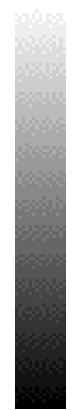

Diffusivity $=$ $0.0025 \mathrm{~cm}^{2} / \mathrm{sec}$

Figure 6. Composite panel thermal diffusivity images with fixed window averaging and variable window averaging.

the number of frames grabbed were 670 for a total inspection time of 11.167 seconds. The PCA analysis was performed by processing images $400-670$. The various compression routines were applied to the data before PCA data reduction. The PCA data reduction was used to compute the first eigenvector image. Shown in Figure 8 are the RCC panel PCA images without compression and sub-sampled every $8^{\text {th }}$ frame. The sub-sampling PCA image was $88.0 \%$ faster to compute. This is a factor of 8 times faster. Also shown in Figure 8 are the fixed window averaging of 10 frames and variable window averaging PCA images. The fixed window averaging and the variable window average PCA images were $92 \%$ and $96 \%$ faster to compute respectively and took around a few seconds to compute. This is a factor of over 12 times improvement in computational speed. The significant speed increase is due to the inversion of a smaller data matrix.

A horizontal line plot was generated over the deepest defect (around image line 90). The line plots represents the average of 5 horizontal lines and this is shown in Figure 9. From the averaged line plot, an average SNR value for the deepest defect was calculated by taking the absolute value of the pixels over the defect minus the average of the background pixels and dividing by the standard deviation of the background pixels. The background pixels were obtained by averaging pixels before and after the defect. The averaged background value was subtracted from the defect value before dividing by the background standard deviation to minimize the effects of the non-flat background. The defect averaged signal to noise values were $1.59,1.43,1.57$, and 1.57 for the non-compressed, sub-sampled every $8^{\text {th }}$ point, 10 point fixed window average, and variable window average PCA images respectively. There is significant degradation in the SNR for the sub-sampled data set and very little degradation for the averaging compression schemes. The computational speed gained is significant for the averaged data.
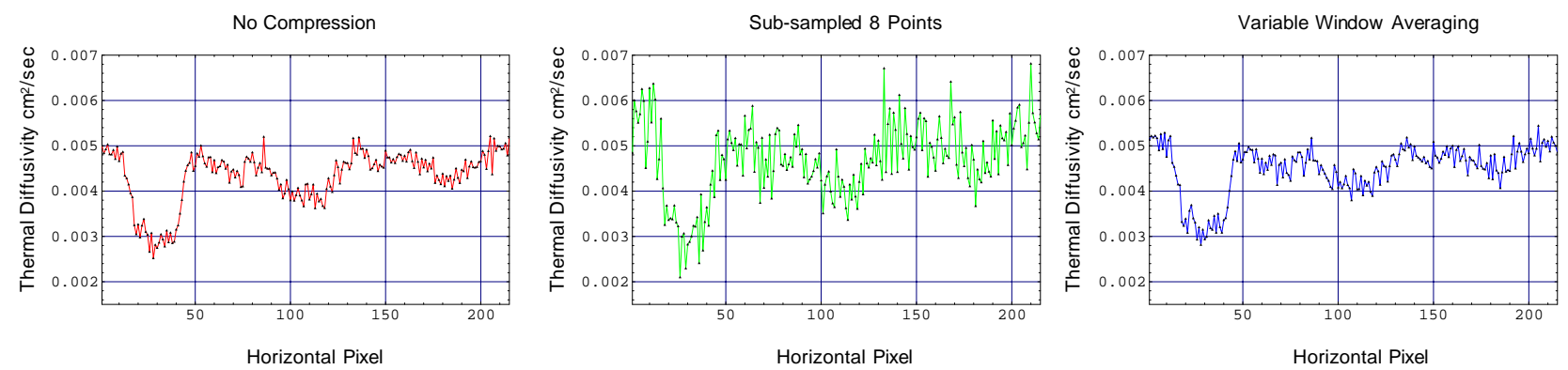

Figure 7. Comparison of horizontal line plots over the first row of defects. 


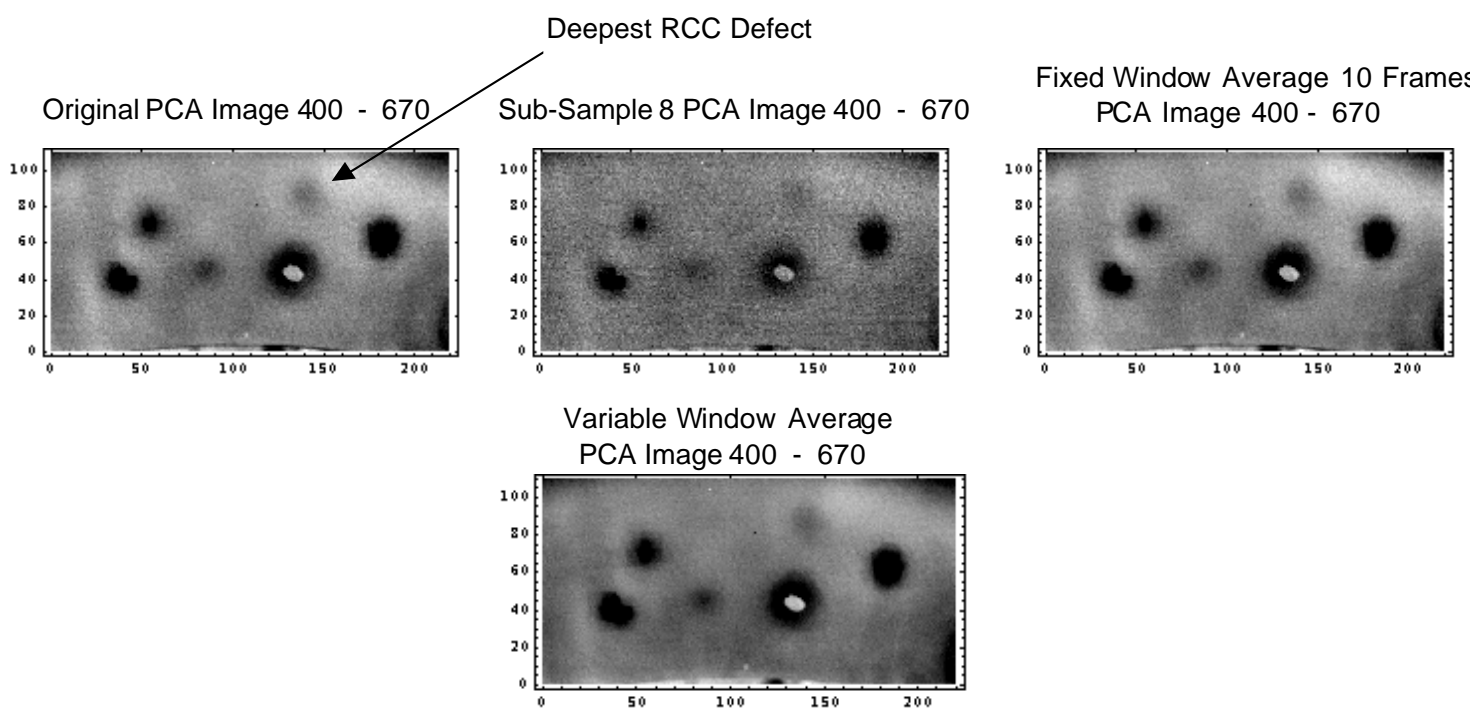

Figure 8. Comparison of PCA images for frames 400-670.

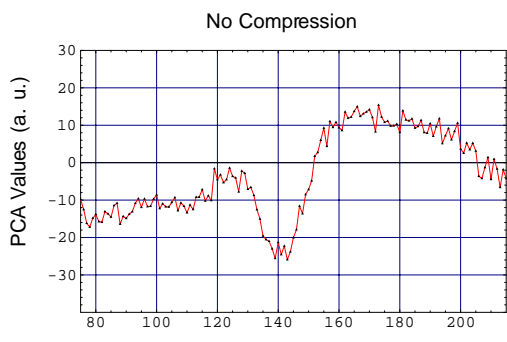

Horizontal Pixel

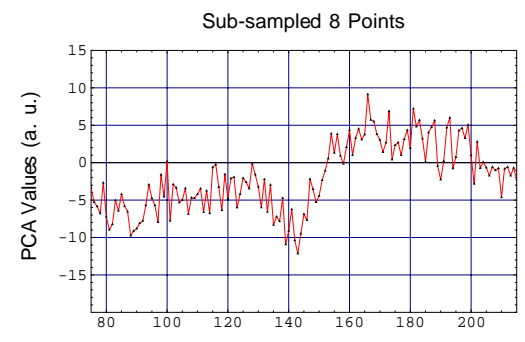

Horizontal Pixel

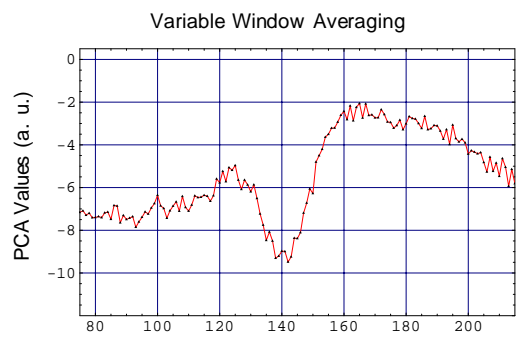

Horizontal Pixel

Figure 9. Comparison of PCA horizontal line plots over the deepest RCC defect.

\section{CONCLUSIONS}

It has been shown that by applying a temporal compression technique, improved algorithm performance can be obtained. For the thermal diffusivity images, there is significant degradation in the SNR for the sub-sampled data set as expected and very little degradation for the averaging compression schemes. The fixed window averaging and the variable window average diffusivity images provided a factor of 2 computational speed increase with little loss in defect contrast. The computational speed gained is even more significant when using the PCA analysis for the fixed window and variable window averaged data. The significant speed increase is due to the reduced time required to invert a smaller data matrix. There was also little SNR degradation in the PCA images when using both the fixed and variable window averaging compression schemes. The variable window averaging routine would be more practical since the compressed thermal data would more suited for defect depth estimation since the early time history is preserved. 


\section{REFERENCES}

1. W. P. Winfree and D. M. Heath, "Thermal diffusivity imaging of aerospace materials and structures", Proceedings of SPIE, Thermosense XXIII, Vol. 3361. 1998, pp. 282-290.

2. N. Rajic, "Principal Component Thermography for Flaw Contrast Enhancement and Flaw Depth Characterisation in Composite Structures", Composite Structures, Vol. 58, pp 521--528, 2002.

3. J. N. Zalameda, "Heat Source Finite Pulse Time Effects in Thermal Nondestructive Evaluation," Materials Evaluation Journal, American Society for Nondestructive Testing, Vol. 60, Nos.3, March, 2002, pp.425-429. 